\title{
Réflexions sur la bona fides et la mala fides dans la tradition du droit romain
}

\author{
L. Waelkens
}

Full professor of Roman law, University of Leuven, Division for Roman Law and Legal History, Sint-Michielsstraat 6 - bus 3453, 300o Leuven, Belgium

Laurent.waelkens@kuleuven.be

\section{Summary}

Good faith has often been discussed in the science of Roman law, from the emergence of fides publica in the Early Republic until the generalisation of good faith in all obligations in the Early Modern continental ius commune. In this article, we stress that it had no religious dimension in Antiquity. A judgment $e$ bona fide meant that the judge had to take all former relations between litigants in account. The association of bona fides with Christian faith and ethics is due to the medieval canonists. The continental generalisation of good faith as applying to all obligations was a consequence of the nationalisation of the consistory courts by the monarchs of the Early Modern Times.

\section{Keywords}

bona fides - good faith - reception of Roman law of obligation - reception of canon law in Early Modern Times

Les romanistes ont souvent abordé la notion de bonne foi. Elle a donné lieu à d'importants développements dans le droit du Moyen-Âge et des Temps Modernes. Depuis le seizième siècle toutes les obligations doivent être exécutées de bonne foi, du moins sur le continent européen. Selon les canonistes la mauvaise foi, la mala fides, nuit à la prescription acquisitive. En effet, ces notions de

* Texte d'une intervention à la Faculté de droit de l'Université de Mons tenue le 27 février 2014. 
bona fides et de mala fides sont issues toutes deux du droit romain. Comment? Notons dès le premier abord qu'elles prennent leur source dans le droit romain de l'Antiquité, mais que leur dimension éthique date de bien après.

Le mot fides apparaît dans les textes de l'ère républicaine. La fides publica y aurait constitué une notion de droit public. Cette fides apparaît dès les premières sources du droit civil, notamment dans le terme bona fides. On la retrouve dans l'Édit Perpétuel ${ }^{1}$ et dans les Institutes de Gaius ${ }^{2}$ à propos de la procédure civile, plus précisément dans les actions et les décisions e bona fide. Ces dernières ne sont pas limitées au droit civil républicain. À l'époque d'Hadrien et par le biais de l'Édit Perpétuel, les actions e bona fide ont été transposées dans la jurisprudence impériale. Hadrien supprima les dernières structures républicaines des provinces sénatoriales et généralisa le régime des provinces impériales. Afin de perpétuer le droit civil de la République, il transplanta l'édit prétorien dans la jurisprudence de la préfecture du prétoire ${ }^{3}$. Ainsi la bona fides républicaine reprit son souffle dans le droit impérial. Elle est présente dans le Corpus iuris du sixième siècle. Elle sera dès lors étudiée aux facultés de droit médiévales et recevra sa place au sein du droit civil occidental.

En 2001 cette notion de bonne foi issue du droit romain a été analysée de façon approfondie dans les journées d'études tenues en l'honneur d'Alberto Burdese à Padoue, Venise et Trévise. Les actes de ce congrès, édités par Luigi

1 O. Lenel, Das edictum perpetuum, Ein Versuch seiner Wiederherstellung, 3e éd., Leipzig 1927 (réimpr. Aalen 2010), passim.

2 Gaius, Institutes, 4,62.

3 Voilà une expression de notre vieille hypothèse sur la composition des Institutes de Gaius. Gaius vivait en Orient et n'y a jamais connu d'autre loi que le droit impérial régi par la jurisprudence du préfet du prétoire. Dans les années 130, l'empereur Hadrien supprima le statut des provinces sénatoriales et les conforma au régime des provinces impériales. Au regret de certains de voir se perdre le droit civil prétorien de la République qui avait fait la grandeur de Rome, il répondit par une nouvelle rédaction de l'édit du préteur. Il fit rédiger par la préfecture du prétoire l'Édit perpétuel afin de l'intégrer dans la jurisprudence impériale. Au début des années 140, Gaius ne composa pas en Orient un cours sur le droit prétorien révolu de la Ville, mais résuma les problèmes créés par l'introduction de l'Édit perpétuel dans le droit impérial. Cette hypothèse explique pourquoi Gaius suit de si près l'Édit perpétuel et pourquoi il énumère tant de controverses et de pièges à éviter. Elle explique aussi pourquoi aucun traducteur qui y voyait de simples règles juridiques n'a satisfait les romanistes. Le mythe selon lequel Gaius a écrit un cours fut basé sur deux éléments. Il y eut d'abord l'affinité avec les simplistes Institutes de Justinien apparemment inspirées de son schéma et dont l'introduction accentue le rôle éducatif. Il y a ensuite le fait que, à la recherche d'un code civil fondé dans l'histoire commune des nations allemandes issues du Congrès de Vienne, les juristes allemands s'extasièrent devant cette nouvelle source. Y voir un simple manuel leur permit de projeter toute une série des principes du code civil français dans leur propre histoire du droit. 
Garofalo en quatre beaux volumes, comportent le texte de quatre-vingt-dix interventions ${ }^{4}$. Ces études constituent une excellente plateforme pour aborder le sujet de la bonne foi dans le droit romain. Elles rendent compte de l'état de la question de la bonne foi dans le droit civil occidental, de l'Antiquité aux temps présents.

\section{$2 \quad$ La bona fides du droit romain antique}

Un bon nombre d'articles de ce recueil concernent le développement de la bona fides dans le droit romain antique. On y retrouve le résumé de toutes les études précédentes vouées au sujet. Malgré la qualité de ces articles synoptiques, il reste difficile d'y saisir exactement ce que signifiait la bona fides antique. Plusieurs intervenants au congrès Burdese ont évoqué son origine religieuse. Une explication d'institutions par la religion correspond rarement à la précision que les juristes recherchent. Chez les romanistes l'évocation de la religion romaine joue même souvent le rôle d'une clause d'exonération. On y contourne élégamment ce qu'on a du mal à comprendre. Dans l'étude fondamentale que Kurt Latte a consacrée à la religion romaine, il ne nous explique pas quels principes de vie elle inculquait aux Romains, mais nous apprend que leur religion aurait surtout été cultuelle et extériorisée ${ }^{5}$. On y retrouve d'anciens vocables des juristes, comme religio, sacrum ou sanctus, divus ou divinus, pius, templum, pontifex ou sacerdos, qui ont été récupérés ensuite par les chrétiens ${ }^{6}$. Est-il permis de projeter leur signification plus récente dans les an-

4 L. Garofalo (éd.), Il ruolo della buona fede oggettiva nell'esperienza giuridica storica e contemporanea, Atti del Convegno internazionale di studi in onore di Alberto Burdese, 4 vol., Padoue 2003; une excellente étude regroupant beaucoup de sources du droit romain de l'Antiquité se trouvait déjà chez L. Lombardi, Dalla fides alla bona fides, Milan 1962.

5 K. Latte, Römische Religionsgeschichte, [Handbuch der Altertumswissenschaft, v,4], Munich 1960.

6 Nous rappelons ici comment nous avons compris ces vocables dans des publications antérieures. Dans les Lois royales et les Douze Tables et toujours chez Cicéron, religio gagne en signification si on le comprend comme « enterrement »; le sens net de « religion » ne se retrouve qu'à partir des Pères latins de l'Église. Sacer («à sanctionner en droit ») et sanctus («sanctionné en droit») sont des adjectifs imparfait et parfait de sanciri, le verbe juridique

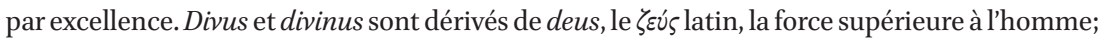
en latin le mot perdit son vocatif en tant que traduction de Jahvé, dont on ne prononçe pas le nom; dans le droit romain antique bien des personnes et des choses étaient supérieures au commun des mortels et à ne pas mettre en ballottage - elles n'en devinrent pas pour autant transcendantales comme le Dieu judéo-chrétien. Antoninus Pius n'était pas pieux, mais intouchable comme une pia causa, un acte juridique irréversible. Il est plus simple de voir dans 
ciennes sources romaines? On peut se poser cette question à propos d'intervenants au congrès Burdese qui invoquèrent l'origine religieuse de la bonne foi romaine. N'ont-ils pas projeté des convictions des Temps Modernes dans l'Antiquité? Peut-être auraient-ils dû apporter des arguments. Expliquer la bonne foi par la religion peut constituer donc un élégant déni de justice. D'autres savants prétendent dans ces études que la fides, tout en ne se référant pas à la religion, fait appel à des valeurs éthiques romaines, des qualités morales. Ils recoupent l'idée des historiens que les Romains cultivaient fides, pietas, virtus, majestas, gravitas ...7 . Là aussi il faut rester prudent. Nous ne connaissons pas de moralistes d'avant les Pères de l'Église. La pensée en valeurs est typique des philosophes 'réalistes' du Moyen-Âge. Chez les juristes médiévaux on constate une émergence de ce réalisme au douzième siècle. Dans la seconde moitié de ce siècle, des mots latins exprimant des relations d'entregent, comme dominium, possessio, matrimonium, obligatio, fiducia, universitas ... reçoivent une dimension réelle et matérielle et commencent à renvoyer à une réalité presque tangible 8 . Mais de là à y découvrir des valeurs éthiques, il y a un pas à ne pas faire. En général, dans l'histoire du droit occidental on ne trouve pas de jugements basés sur des principes éthiques avant la scolastique espagnole de la seconde moitié du seizième siècle.

En abordant le latin de l'Antiquité et contrairement à ce qu'on fait lorsqu'on lit des textes universitaires du Moyen-Âge, il ne faut pas chercher en premier lieu les valeurs exprimées par le sujet, le verbe ou le complément d'objet. Il faut d'abord ressentir les relations personnelles exprimées par la phrase. Avec un nombre invraisemblable de formes du verbe et de pronoms personnels les Romains cherchaient à exprimer en premier lieu des relations entre personnes. Voilà une caractéristique de la linguistique latine. Ces relations se retrouvent également exprimées dans les substantifs. Par piété, majesté, discipline,

un antique templum un lieu de justice qu'un lieu de culte. Pontifex et sacerdos étaient des juges du ius sacrum, la procédure remplacée par les régimes civil et impérial; les juifs et chrétiens utilisaient ces termes pour leurs rabbins et évêques, qui étaient autant juges que prêtres. À chaque fois, les nouvelles significations de ces mots ne sont pas à projeter dans les premières périodes du droit romain.

7 Comme nous le rappellent ailleurs B. Linke et M. Stemmler, Mos maiorum, Untersuchungen zu den Formen der Identitätsstiftung und Stabilisierung in der römischen Republik, Stuttgart 2000.

8 L'entrée du néoplatonisme dans l'œuvre des juristes est remarquable chez les canonistes parisiens de la seconde moitié du douzième siècle dont les œuvres ont été éditées dans le Monumenta iuris canonici, Series A: Corpus glossatorum, Cité du Vatican. La conceptualisation du droit, absente chez Gratien, se trouve dans la Summa lipsiensis et devient frappante dans les gloses de magister Honorius. 
gravité ... les Romains exprimaient de bonnes relations entre les gens. Il est assez probable que la fides aussi ait exprimé une entente. Dans l'Antiquité et jusqu'aux Temps modernes le mot fides a recoupé le mot grec $\pi i \sigma \tau \iota s$ et le verbe fidere s'est retrouvé associé avec $\pi \varepsilon i \vartheta \varepsilon \nu^{9}$. Ces mots renvoient à la discussion, à la persuasion, à l'argumentation. En Grec les formes médiales et passives de $\pi \varepsilon i \vartheta \varepsilon \iota \nu$ sont utilisées pour invoquer les suites de la persuasion ou d'un accord, l'issue de pourparlers, l'acceptation d'une entente, la confiance dans la solution et l'engagement de s'y tenir. La fides n'aurait-elle rien eu affaire avec des pourparlers et ententes? Lewis et Short nous signalent que fides est un synonyme de foedus. Fidere a affaire avec un traité ou un accord susceptible d'établir la confiance. Du verbe on passe au substantif. Nuire à la fides n'est pas un outrage à une attitude bienveillante, mais consiste à ne pas respecter un accord. Les plus anciennes occurrences de fides et $\pi i \sigma \tau \iota \zeta$ se trouvent dans fides publica et $\pi i \sigma \tau \iota \zeta \delta \eta \sigma^{\sigma} \sigma \alpha$. Elles concernent les pourparlers et les traités de paix ${ }^{10}$. Dans la belle étude que G. Freyburger a consacrée à la fides romaine, nous retrouvons ce renvoi à la confiance ${ }^{11}$. Bien des savants y voient une vertu ou une norme générale ${ }^{12}$. Pourtant, la fides publica n'indique pas l'état d'âme des dirigeants de l'armée, mais le traité lui-même. Au Capitole l'aedes fidei était le bâtiment où les traités étaient signés, conservés et parfois affichés ${ }^{13}$.

La fides qui apparaît dans le droit civil fait référence à la même entente. L'Édit perpétuel et Gaius mentionnent des actions dont la formule contient les mots e bona fide: la vente et l'achat, la location, la société, le mandat, la fiducie,

Comparez à ce propos les mots fides et fidere chez Lewis and Short, A Latin Dictionary, avec $\pi i \sigma \tau \iota$ et $\pi \varepsilon i \vartheta \varepsilon \imath \nu / \pi \varepsilon i \vartheta_{0} \mu \alpha \iota$ chez Liddell and Scott, Greek-English Lexicon. Le mot grec $\pi \iota \sigma \tau \varepsilon \varepsilon \varepsilon i v$, avoir confiance, a été déduit ensuite de $\pi i \sigma \tau \iota \varsigma$. Cf. les considérations développées par É. Benveniste, Le vocabulaire des institutions indo-européennes, Paris 1969, I : Économie, parenté, société, p. 103-121.

10 Voir par ex. l'article de D. Nörr, 'Fides Punica - Fides Romana', Bemerkungen zur 'demosia pistis' im ersten karthagisch-römischen Vertrag und zur Rechtsstellung des Fremden in der Antike, in: La buona fede oggetiva (supra, n. 4), II, p. 497-549. Cf. D. Nörr, Die fides im römischen Völkerrecht, Heidelberg 1991.

11 G. Freyburger, Fides, Étude sémantique et religieuse depuis les origines jusqu'à l'époque augustéenne, Paris 1986. Contrairement à Benveniste, Vocabulaire des institutions (supra, n. 9), Freyburger cherche à donner une dimension religieuse à la fides. Il analyse le culte de la main droite, symbole de la confiance.

12 Ainsi M. de Wilde, 'Fides publica' in Ancient Rome and its reception by Grotius and Locke, Tijdschrift voor Rechtsgeschiedenis, 79 (2011), p. 461, qui en fait une norme générale. Il résume toute la bibliographie qui va dans ce sens.

13 Sur ce bâtiment voir Chr. Reusser, Der Fidestempel auf dem Kapitol in Rom und seine Ausstattung, Rome 1993. L'a. décrit les fouilles et l'histoire du bâtiment, mais fait de ce dernier une sorte d'église dédiée par des Romains croyants à Sainte Foy. 
la tutelle, la gestion d'affaires, le prêt et la mise en dépôt. D'autres actions suivraient: l'action dotale, le commodat et l'action servienne. Une procédure entamée à partir de l'une de ces actions, conduisit à un iudicium bonae fidei. En effet, le juge qui devait statuer sur une action $e$ bona fide, était tenu de vérifier toutes les relations entre les litigants. Il tenait compte des conventions accessoires, de pactes informels, de l'exécution d'obligations antérieures, des circonstances ayant amené l'obligation, de dol, de contrainte ... ${ }^{14}$. Dans les procès introduits à partir d'une action où les mots $e$ bona fide ne figuraient pas, le juge ne vérifiait pas tous ces éléments. Ainsi par exemple lorsqu'une obligation stricte faisait l'objet d'un litige, le juge ne pouvait contrôler que la régularité de l'acte juridique ayant engendré l'obligation. Si ce dernier était valable, le défenseur était condamné; si l'acte était invalide, il était libéré. Dans les sentences de bonne foi (iudicia bonae fidei) le juge évaluait donc toute la relation entre les parties et condamnait à payer ce qui était dû $e$ bona fide, à cause des exigences d'une bonne relation. Sous l'empire, la jurisprudence élargit dans certaines conditions les sentences $e$ bona fide aux actions strictes. Elle le fit par l'insertion de l'exception de dol dans les actions strictes ${ }^{15}$. Pour obtenir celle-ci le défendant dut prouver au tribunal, lors de la session d'introduction, que le plaignant était un homme de peu de confiance. L'insertion d'une exception de dol dans une action obligea le juge de fond à prononcer un jugement $e$ bona fide, où tous les aspects de la relation entre les parties jouaient leur part. Dans le droit impérial romain contenu dans le Corpus iuris, on ne trouve aucune autre bona fides que cette bonne relation entre les parties ${ }^{16}$. Rappelons que la fides catholica qui se trouve dans le titre 1,1 du Code de Justinien y a été ajoutée au douzième siècle ${ }^{17}$.

14 M. Kaser, Das Römische Privatrecht, I, Munich 1971, p. 486-488; II, Munich 1973, p. 333-335; pour l'action dotale voir F. Goria, 'Bona fides' ed 'actio ex stipulatu' per la restituzione della dote: legislazione giustinanea e precedenti classici, in: La buona fede oggetiva (supra, n. 4), II, p. 241-263.

15 Matière résumée dans D. 44,4.

16 Voir les références chez M. Kaser, Römisches Privatrecht, en n. 15; cf. H.G. Heumann et E. Seckel, Handlexicon zu den Quellen des römischen Rechts, Graz 1958, s.v. bona fides; M. Schermaier, 'Bona fides' im römischen Vertragsrecht, in: La buona fede oggetiva (supra, n. 4), III, p. 387-416.

17 L. Waelkens, L'hérésie des premiers titres du Code de Justinien, Une hypothèse sur la rédaction tardive de C. 1,1-13, Tijdschrift voor Rechtsgeschiedenis, 79 (2011), p. 253-296; hypothèse contestée par B.H. Stolte, A heretical hypothesis, On the beginning of the Codex Justinianus, Tijdschrift voor Rechtsgeschiedenis, 81 (2013), p. 109-128. 
La fides catholica apparut cependant plus tôt dans les écrits des Pères de l'Église. Dans les premiers siècles de notre ère, ils utilisèrent les mots grec $\pi i \sigma \tau \iota \varsigma$ et latin fides pour indiquer la 'nouvelle alliance' des chrétiens. Dans la patristique ces termes passèrent progressivement de la discipline à la doctrine et se mirent à indiquer l'ensemble de la pensée chrétienne. Dans le dictionnaire de Lampe, où $\pi i \sigma \tau \iota \zeta$ remplit neuf colonnes, on remarque comment à travers les auteurs chrétiens grecs la signification du mot passe progressivement de la nouvelle alliance à l'ensemble du credo de Nicée ${ }^{18}$. Les théologiens romains ont également fait appel à la terminologie du droit romain pour établir leur vocabulaire chrétien ${ }^{19}$. Fides resta la traduction latine de $\pi i \sigma \tau \iota \zeta$ même quand la signification de ce dernier mot passa de l'alliance à la croyance. Chez Saint Augustin la bona fides signifie toujours loyauté, mais il trouve que celle-ci est nécessaire dans l'exécution de tous les actes juridiques ${ }^{20}$. Au cinquième siècle Innocent Ier décrira également la nécessité de respecter le droit impérial avec bona fides, avec loyauté. Chez Saint Augustin et Innocent $\mathrm{I}^{\mathrm{er}}$ cette loyauté était déjà chargée de principes chrétiens comme le respect de la société, la charitas et l'amour du prochain. Dans le Bréviaire d'Alaric, la fides est devenue fidélité. Au cours du sixième siècle, les rois visigotiques associèrent le respect du droit avec la discipline chrétienne ${ }^{21}$. Le mot latin fides gagnerait l'Occident comme « foi » ou « faith », des mots qui deviendraient des vecteurs du christianisme.

Les canonistes médiévaux ont ensuite développé l'idée de la bona fides généralisée dans le droit. Dans le Décret de Gratien de 1140, fides tout court renvoie à la foi chrétienne, à la fidélité et à la fiabilité2 ${ }^{2}$. Dans deux passages du Décret la bona fides est mentionnée comme principe général de droit: on doit administrer les biens de l'église avec grande diligence et « avec la bonne foi qu'on doit à Dieu »23; les serfs doivent servir leur patron de bonne foi ${ }^{24}$. La bonne foi y exprime toujours les bonnes relations, mais l'accent est passé ici de

18 G.W.H. Lampe, A patristic Greek lexicon, Oxford 1961.

19 Voir les exemples chez J. Gaudemet, Le droit romain dans la littérature chrétienne occidentale du IIIe au Ve siècles, [Ius romanum medii aevi, I,3,b], Milan 1978.

20 R. Rodriguez Lopez, La 'bona fides' en los textos cristianos, in: La buona fede oggetiva (supra, n. 4), III, p. 255-277, p. 264 sqq. Voir l'excellent article de E. Osaba, Fides y bona fides en la lex visigothorum, in: La buona fede oggetiva (supra, n. 4), II, p. 543-778.

22 On peut facilement contrôler des vocables dans le Décret de Gratien en utilisant le site $<$ http://geschichte.digitale-sammlungen.de/decretum-gratiani/>.

23 C. 10 qu. 1 c. 5 .

24 C. 17 qu. 4 c. 37. 
l'alliance à la charitas chrétienne de Saint Augustin. Cent ans plus tard, dans ses commentaires sur les Décrétales de Grégoire IX, l'Hostiensis laisse transparaître dans le droit canon une théorie cohérente de la bonne foi généralisée. Bona fide semper agendum est et animarum periculis occurendum, il faut toujours agir de bonne foi et tenir compte des dangers qu'encourt lâme ${ }^{25}$. 'Bona fides dicitur esse in eo qui habet bonam conscientiam', la bonne foi anime celui qui a bonne conscience ${ }^{26}$. 'Bona fides et dolus contraria sunt', la bonne foi et le dol sont des éléments contraires ${ }^{27}$. 'Bona fides est consideranda in omni contrac$t u$ ', la bonne foi est à respecter dans tout contrat ${ }^{28}$. Elle ne se limite cependant pas aux contrats: 'bona fides non patitur quod bis idem exigatur', la bonne foi ne permet pas que la même chose soit réclamée deux fois ${ }^{29}$. La bonne foi était devenue une attitude. Elle serait développée par les canonistes en une disposition de l'âme, en vertu de laquelle l'auteur d'un acte se conforme à la loi morale ou aux prescriptions ${ }^{30}$. Les canonistes ne quitteraient plus l'idée que cette bonne foi était nécessaire dans tous les actes juridiques. Ils la défendirent et la développèrent aussi longtemps qu'ils gardèrent une influence sur la jurisprudence, c'est-à-dire du douzième au dix-septième siècle ${ }^{31}$.

Les canonistes ont également introduit dans le droit, par leur enseignement du droit pénal, les critères du bien et du mal, de l'intention et de la faute morale $^{32}$. Malgré le fait que les canonistes n'aient jamais eu de compétence dans le droit pénal, leur enseignement de cette branche a fondamentalement influencé les catégories du droit romain ${ }^{33}$. Ils ont également intériorisé la bona fides, l'ancien principe de la bonne entente. Ceci est illustré par le développement de la mala fides, une expression qui figurait au Corpus iuris et y signifiait «sans bonne entente ${ }^{34}$. Les canonistes en ont fait une notion éthique, qu'ils

25 Hostiensis, commentaire sur X. 2,10,2 (éd. Venise 1581, fol. 37 rb n. 35).

26 Id., sur X. 2,26,17 (éd. Venise 1581, fol. 154 ra n. 8).

27 Id., sur X. 3,16,2 (éd. Venise 1581, fol. 57 ra, n. 1).

28 Id., sur $\mathrm{x} .3,17,3$ (éd. Venise 1581 , fol. 59 ra n. 8).

29 Id., sur X. 3,30,26 (éd. Venise 1581, fol. 103 ra n. 12).

$30 \quad$ F. Claeys-Bouuaert, Bonne foi, in: Dictionnaire de droit canonique II, Paris 1937, col. 957.

31 Id., col. 96o-961.

32 Voilà le sujet de l'étude inégalée de S. Kuttner, Kanonistische Schuldlehre von Gratian bis auf die Dekretalen Gregors IX., Systematisch auf Grund der handschriftlichen Quellen dargestellt, Cité du Vatican 1935.

33 Cf. L. Waelkens, Et si la responsabilité pénale du droit romain datait du douzième siècle?, in: J.-M. Carbasse et M. Ferret-Lesné (éd.), Doctrines et pratiques pénales en Europe, Montpellier 2012, p. 87-95.

34 Voir par ex. D. 17,2,3,3; D. 41,1,48,1; D. 41,3,38; clairement dans D. 5,3,25,7: toute possession est mala fide de plein droit après la litiscontestatio; la mala fides est donc procédurale. 
ont introduite dans le droit civil de la prescription lorsque celle-ci était discutée devant les officialités. Cette mala fides éthique se découvre dans les Décrétales de Grégoire VII, dans X. 2,26,20: elle empêche la prescription acquisitive. Au Moyen-Âge le dominium des terres agricoles tombait sous la compétence des cours féodales et toute transmission était contrôlée par celles-ci. Certains exploitants et les abbayes évitèrent cette instance en tenant leurs terres en possession $^{35}$. Les possessions restèrent en-dehors du régime féodal et devinrent des «alleux ». Dans le droit romain antique la possession s'obtenait par une mesure provisoire du tribunal. En cas d'un conflit au sujet de l'attribution d'un terrain, les quérulants pouvaient requérir un interdit, une mesure possessoire. Ils avaient cette possibilité pendant un an, à compter à partir des premiers faits faisant preuve de leur différend. La mesure possessoire était établie par un juge, et bien e bona fide, de façon contradictoire, après discussion entre les prétendants au terrain. Elle devenait définitive par la prescription extinctive (après dix ou vingt ans) de la revendication de ceux qui étaient lésés par l'interdit. Cette procédure possessoire a été amendée au Moyen-Âge. Afin de rester à l'écart de la justice féodale, les juristes ont adapté les règles romaines. Après le Concordat de Worms (1122) les juges ecclésiastiques devinrent compétents pour les biens des abbayes et pour les alleux aux mains des évêques. Pour eux l'acquisition du bien se faisait par une custodia, une tenure extra-judiciaire, pendant un an, le temps dont les autres disposaient pour intenter l'interdit. Après un an et un jour, la possession était établie. Dans la gl. 'Etiam actionem' ad C. 7,37,8pr. nous apprenons que cette possession n'était plus une mesure provisoire, mais que, à l'instar de la revendication pour le dominium, elle était également exigible au moyen d'une action possessoire. La custodia de la première année n'étant plus établie de façon contradictoire, on pouvait mettre en doute qu'elle fut bien établie e bona fide. Dans X. 2,26,20 les canonistes renversèrent la situation: pendant cette première année il ne pouvait y avoir de mala fides. Dans cette interprétation de la fides, on constate qu'une notion de procédure était devenue une notion matérielle, une attitude éthique: le détenteur du bien ne pouvait avoir lésé intentionnellement les droits d'autrui sur le bien en question.

Chez les canonistes et dans les officialités, la bona fides ferait partie de toutes les obligations ${ }^{36}$. En effet, en tant que fides, elle fut associée à la charité chrétienne. Même les marchands les plus durs ne pouvaient manquer à leur devoir de charité. Cette bona fides des canonistes ne se retrouve pas chez les légistes.

Le système est très ancien: voir J. Durliat, Les finances publiques de Dioclétien aux Carolingiens (284-889), Francia, Beihefte, 21], Sigmaringen 1990, passim.

36 Le locus communis de cette idée se trouve dans la gl. 'Bonae fidei' ad x. 2,25,6. 
Aux facultés de droit on continua à distinguer clairement entre les actions de bonne foi et les actions strictes. Personne ne suggéra que ces dernières manquaient de sérieux ou que les actes donnaient lieu à l'iniquité37. Les glossateurs et les commentateurs limitèrent leurs études au Corpus iuris. Ils n'y trouvaient pas cette bonne foi étrangère au droit romain, cette valeur à respecter, cette attitude à adopter. Vers la fin du treizième siècle le légiste Pierre de Belleperche, qui associa bona fides et absence de dol, fut le premier à mettre en doute la distinction entre obligations de bonne foi et obligations strictes. Il eut peu de répondant. Cinus de Pistoia signala son point de vue, mais Bartole accentua toujours l'importance des obligations strictes et l'importance de ces actions romaines qui n'exigeaient pas de jugement de bonne foi ${ }^{38}$. Les grands commerçants utilisaient couramment les stipulations orales, les novations, les stipulations écrites, toutes des moyens sûrs et négociables. Encore au quinzième siècle on remarque l'approche différente des canonistes et des tribunaux ecclésiastiques d'une part, qui promurent la bonne foi généralisée, et de l'autre les légistes, les tribunaux des villes et les conseils souverains qui limitèrent la bonne foi aux iudicia bonae fidei énumérés dans le Corpus iuris.

\section{$4 \quad$ La réception de cette bona fides par les civilistes}

Aux Temps Modernes la bonne foi des canonistes a gagné le devant de la scène d'une façon inattendue. Partout en Occident les monarques qui avaient introduit le droit impérial dans leurs conseils souverains, ont fini par monopoliser le pouvoir en regroupant toutes les compétences judiciaires au sein de leurs conseils souverains. Dès les années 1530, partout en Europe les justices ecclésiastiques furent soumises à la justice royale. Les évêques scandinaves furent enrôlés comme fonctionnaires royaux et toutes leurs institutions devinrent royales. Dans les pays réformés allemands les institutions ecclésiastiques furent communalisées et leur jurisprudence soumise aux échevinages. Dans les pays catholiques, les princes introduisirent la double compétence et acceptèrent des affaires de droit canon dans la justice royale. Dans les Pays-Bas le champion de cette double compétence fut Nicolas Evrard, président du Grand

R. Zimmermann, The law of obligation, Roman foundations of the civilian tradition, Le Cap

- Wetton - Johannisbourg 1990, passim au sujet des obligations strictes; spécialement p. 807 et suivantes, au sujet du 'breach of contract under the ius commune'.

$3^{8}$ Le résumé de cette discussion se trouve chez W. Decock, Theologians and contract law, The moral transformation of the ius commune (ca.1500-1650), Leyde - Boston 2013, p. 274279 . 
Conseil de Malines ${ }^{39}$. Les conseils souverains étant devenus compétents pour toutes les matières, les juges royaux se mirent à introduire dans leur jurisprudence une série de principes empruntés aux canonistes, comme 'pacta sunt servanda', l'intention à l'origine des obligations ou ... la généralisation de la bonne foi. En effet, étant obligés de traiter des affaires qui auparavant se discutaient devant les juridictions ecclésiastiques, les conseillers souverains firent appel à l'ancienne jurisprudence canonique. Ainsi ils adoptèrent dans leur ius commune la tradition des canonistes et la mélangèrent à celle des civilistes. Pour justifier cette pratique, les civilistes soutinrent que les principes du droit canon faisaient partie intégrante du droit civil européen en tant que droit coutumier ${ }^{40}$.

Parmi ces principes des canonistes, il y avait donc la généralisation de la bonne foi. Dans la seconde moitié du siècle on vit poindre l'idée que celle-ci devait toujours être respectée, fût-ce de façon différente dans les actes juridiques strictes et dans les actions de bonne foi ${ }^{41}$. Au dix-septième siècle le mélange des deux matières deviendrait intime. On vit alors se profiler l'idée exprimée en 1804 dans l'art. 1134, $3^{\circ}$ du Code civil, selon lequel toutes les obligations doivent être interprétées de bonne foi. À partir de cette époque la généralisation de la bonne foi sera ressentie comme une exigence de l'équité ${ }^{42}$. Elle est restée étrangère à la justice anglaise. En effet, en Angleterre Henri viII a bien mis les tribunaux sous le contrôle du Conseil privé, mais les a laissé fonctionner en autonomie. Ainsi le droit anglais royal n'a pas eu l'apport des canonistes et jusquà présent les Anglais éprouvent des problèmes à adopter la généralisation de la bonne foi ${ }^{43}$.

Le poids de l'absolutisme se fit sentir lourdement vers la moitié du seizième siècle et plaça les juristes dans une situation difficile: comment réagir contre un prince absolu? Les juristes avaient remarqué que certains princes ne reculaient que devant Dieu. Ainsi ils se mirent à introduire des principes « divins » dans le droit. En effet, comme premiers les jusnaturalistes espagnols prétendirent que les droits civils étaient «naturels». Le Créateur les avait insufflés à

\footnotetext{
39 Voir L. Waelkens, Nicolas Everaerts, Un célèbre méconnu du droit commun (1463/4-1532), Revista internazionale di diritto comune, 15 (2004), p. 173-183.

40 Thèse du l'étude de U. Wolter, Ius canonicum in iure civili, Studien zur Rechtsquellenlehre in der neueren Privatrechtsgeschichte, Vienne - Cologne - Weimar 1975.

Cujas exprime bien cette idée dans ses Observationes et emendationes, 8,10:In contractibus strictis quemadmodum bona fides exigatur, in: I. Cujaicii Opera quae de iure fecit et edere voluit, Francfort/Main 1623, IV, col. 168-169.

Voir F. Donato Burnelli, Note in tema di buona fede ed equità, in: La buona fede oggetiva (supra, n. 4), I, p. 225-255.

43 Ibid., p. 246, avec bibliographie.
} 
toute homme lors de sa naissance (sa natura). Le prince et les juges souverains avaient intérêt à respecter ces droits naturels et supranationaux s'ils aspiraient à l'au-delà. Ils en devraient répondre au Dernier Jugement. L'accentuation de valeurs extra-juridiques, voir théologiques, dans le but de retirer le pouvoir absolu aux monarques, a fait l'objet d'une étude approfondie par Wim Decock. On y constate que la bonne foi se trouvait également dans les valeurs imposées par l'intermédiaire des théologiens ${ }^{44}$. À partir de cette période de l'histoire du droit romain les civilistes acceptèrent de défendre la bonne foi exprimant une attitude religieuse.

\section{$5 \quad$ Conclusion}

En fait, lorsque les romanistes prétendent que la bona fides du droit romain exprime une valeur traditionnelle, ils ont raison. Toutefois, ils ne se réfèrent pas à une valeur de l'Antiquité, mais aux Temps Modernes. Et ceux qui lient la bona fides à la religion romaine ont-ils également raison? S'ils acceptent que cette religion soit le christianisme ... Par là on en revient à cette conclusion terrible que le droit romain de l'Antiquité tel que l'on l'envisage maintenant n'a jamais existé, qu'il fut inventé par des juristes et des historiens qui relurent les textes antiques avec le ius commune des Temps Modernes en arrière-pensée ${ }^{45}$.

44 W. Decock, Theologians and contract law (supra, n. 38). On peut retrouver au moyen du registre les nombreux passages imposant la pratique de la bona fides.

45 Hypothèse formulée lors d'une intervention à Besançon: L. Waelkens, Le droit romain antique, un produit de l'interprétation des Temps Modernes?, in: B. Barnabé (éd.), L'interprétation, Journées internationales d'histoire du droit, Besançon 30 mai - 1 juin 2013, Besançon (sous presse). 